\title{
Does Oil and Gold Price Uncertainty Matter for the Stock Market?
}

\author{
Dennis Bams - Maastricht University \\ Gildas Blanchard - Maastricht University \\ Iman Honarvar - Maastricht University
}

Thorsten Lehnert - Luxembourg School of Finance 